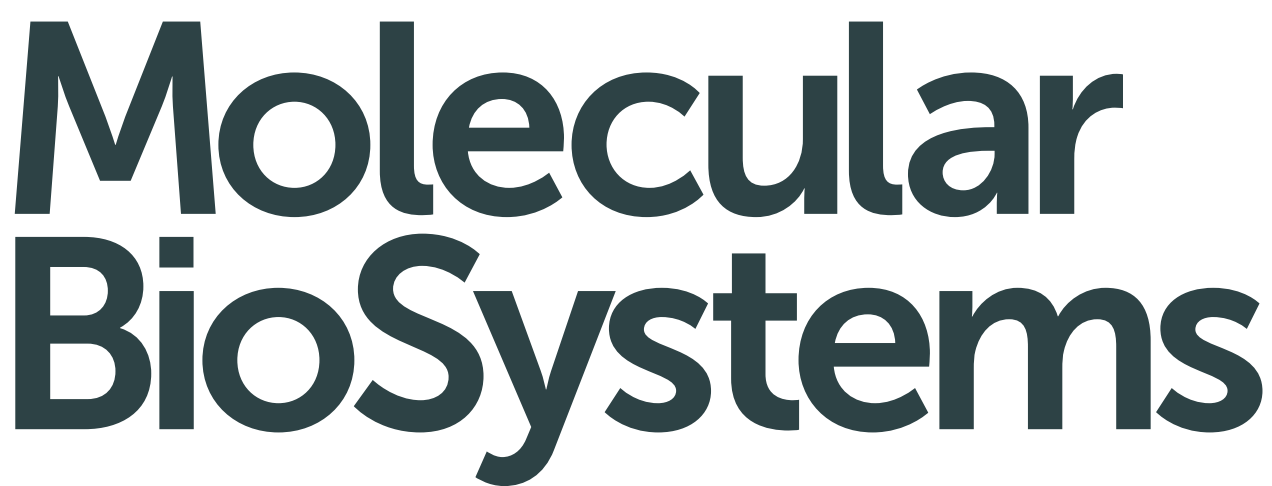

Interfacing chemical biology with the -omic sciences and systems biology rsc.li/molecular-biosystems
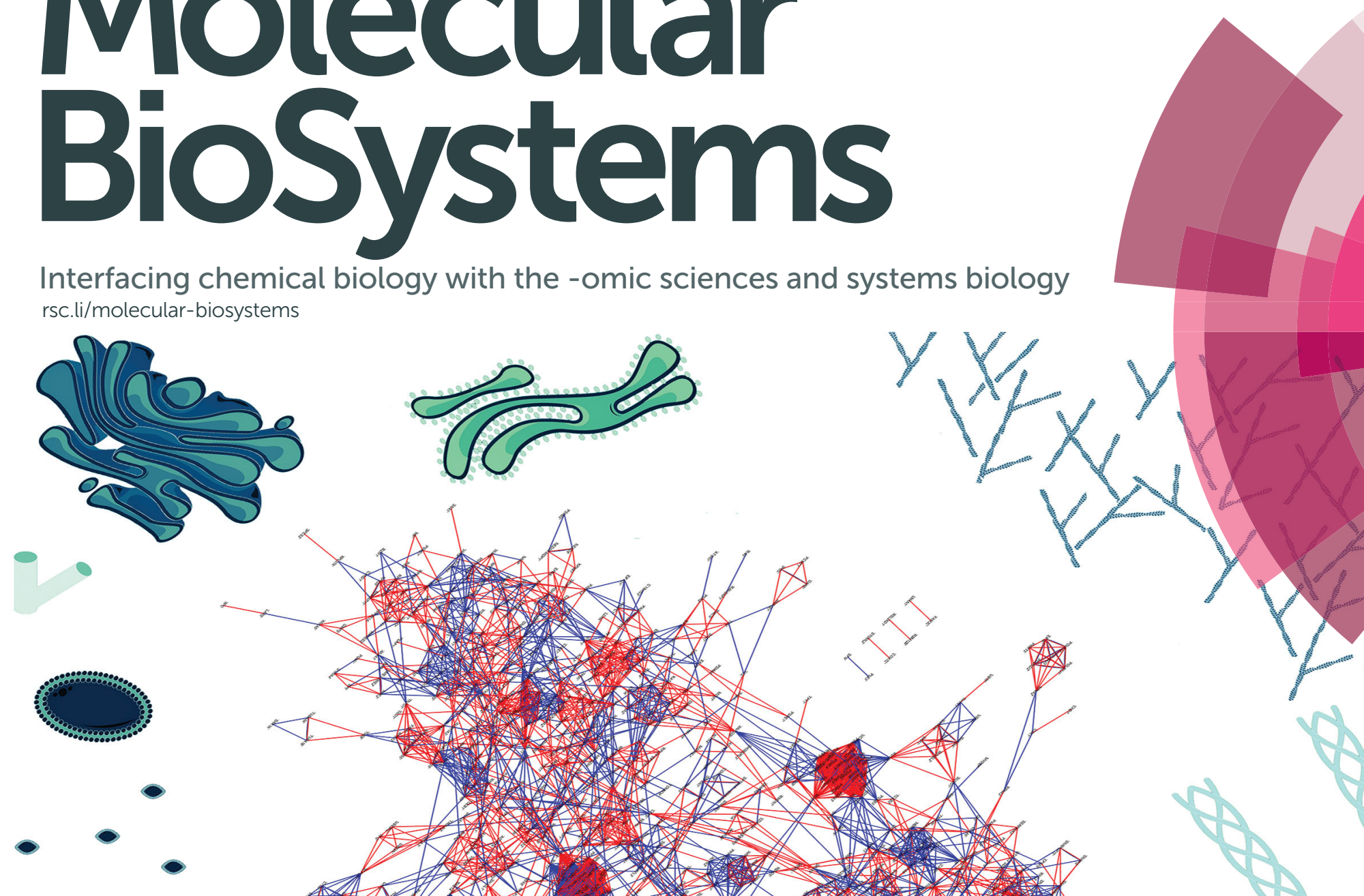

Sins
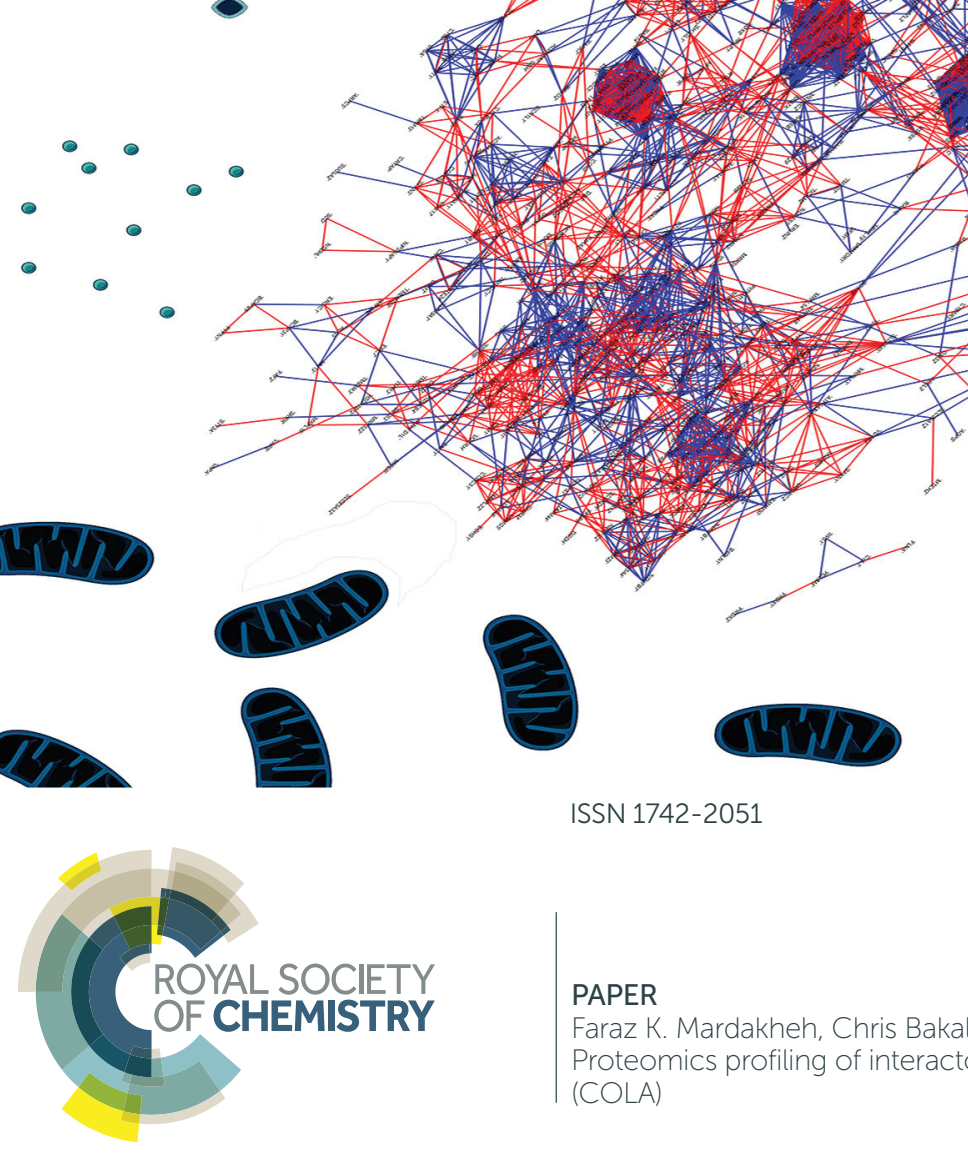


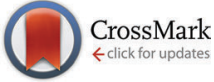

Cite this: Mol. BioSyst., 2017, 13,92

Received 12th October 2016, Accepted 1st November 2016

DOI: $10.1039 / \mathrm{c} 6 \mathrm{mb} 00701 \mathrm{e}$

www.rsc.org/molecularbiosystems

\section{Proteomics profiling of interactome dynamics by colocalisation analysis (COLA) $\dagger$}

\author{
Faraz K. Mardakheh, $\ddagger^{\star^{a}}$ Heba Z. Sailem, $\ddagger^{\text {ab }}$ Sandra Kümper, ${ }^{a}$ Christopher J. Tape, ${ }^{\text {ac }}$ \\ Ryan R. McCully, ${ }^{a}$ Angela Paul, ${ }^{a}$ Sara Anjomani-Virmouni, ${ }^{a}$ Claus Jørgensen, ${ }^{d}$ \\ George Poulogiannis, ${ }^{a}$ Christopher J. Marshall $\S^{a}$ and Chris Bakal ${ }^{* a}$
}

\begin{abstract}
Localisation and protein function are intimately linked in eukaryotes, as proteins are localised to specific compartments where they come into proximity of other functionally relevant proteins. Significant co-localisation of two proteins can therefore be indicative of their functional association. We here present COLA, a proteomics based strategy coupled with a bioinformatics framework to detect protein-protein co-localisations on a global scale. COLA reveals functional interactions by matching proteins with significant similarity in their subcellular localisation signatures. The rapid nature of COLA allows mapping of interactome dynamics across different conditions or treatments with high precision.
\end{abstract}

\section{Introduction}

Systems level understanding of biological processes requires unravelling of functional interactions on a global scale. A functional interaction is a molecular association between two or more proteins which share a common, interdependent, biological function. Mining of such interactions on a global scale is often achieved by high-throughput genetic screens, ${ }^{1}$ where interactions are inferred by commonality in phenotypic outcomes of genetic gain or loss of function events. ${ }^{2,3}$ Alternatively, functional interactions can be inferred from molecular interaction analyses, on the principle of 'guilt by association'. ${ }^{4}$ Determining such interactions on a global scale, however, has remained a fundamental technical challenge. Reporter based methods such as Yeast-2Hybrid ( $\mathrm{Y} 2 \mathrm{H})$ or protein-fragment complementation assay (PCA) are labour intensive, and can only reveal direct biochemical interactions. ${ }^{5,6}$ Affinity Purification coupled with Mass Spectrometry (AP-MS), ${ }^{7,8}$ or proximity based labelling, ${ }^{9}$ are similarly labour intensive, but do reveal indirect interactions. A major downside of both AP-MS and proximity based labelling methods, however, is that the analysis time is directly proportional to the

\footnotetext{
${ }^{a}$ Institute of Cancer Research, Division of Cancer Biology, 237 Fulham Road, London SW3 6JB, UK. E-mail: chris.bakal@icr.ac.uk, mardakheh@icr.ac.uk

${ }^{b}$ Institute of Biomedical Engineering, University of Oxford, Old Road Campus Research Building, Oxford, OX3 $7 D Q$, UK

${ }^{c}$ Department of Biological Engineering, Massachusetts Institute of Technology, Cambridge, Massachusetts 02139, USA

${ }^{d}$ Cancer Research UK Manchester Institute, University of Manchester,

Wilmslow Road, Manchester M20 4BX, UK

$\dagger$ Electronic supplementary information (ESI) available. See DOI: 10.1039/c6mb00701e

\# These authors contributed equally to the work.

$\S$ Deceased.
}

number of proteins being investigated, rendering large scale comparative interactome analysis across different conditions difficult due to an increasing number of mass spectrometry runs. Moreover, tagging of cellular proteins required for both methods could affect their activity, resulting in potential artefacts.

Recently, a novel proteomics based approach has been utilised to reveal interactions by assessing the co-behaviour of interacting proteins when biochemically fractionated. ${ }^{10}$ Havugimana et al. used three parallel chromatography methods in conjugation with proteomics, separating soluble cellular complexes based on charge, $\mathrm{p} K_{\mathrm{a}}$, and density. ${ }^{11}$ Kristensen et al., on the other hand, used size-exclusion chromatography in conjugation with proteomics to separate complexes by size. ${ }^{12}$ Proteins with similar elution profiles were then matched as likely constituents of the same complexes. The key advantage of these approaches is allowing simultaneous determination of biochemical interactions from a fixed number of mass spectrometry runs. ${ }^{12,13}$ However, a downside of both approaches is that they are limited to soluble proteins, and therefore are not well suited for detecting insoluble complexes. Moreover, while matching proteins based on biochemical co-fractionation can reveal strong associations that survive such fractionations, many physiologically relevant functional interactions only occur transiently, thus are often missed during stringent biochemical separations. ${ }^{9}$

Eukaryotic cells are highly compartmentalised assembly of organelles, macro-molecular complexes, and spatially organised subcellular functional regions. As a result, where a protein is localised inside a eukaryotic cell can be indicative of its function. More importantly, colocalisation of two or more proteins can be indicative of their functional interaction. ${ }^{14}$ We hypothesised that, similar to multi-variate phenotypic signatures used in 
high-throughput genetic interaction analyses, ${ }^{2}$ quantitative multi-dimensional subcellular localisation signatures can be used to match functionally interacting proteins on the basis of their colocalisation. We here present COLA, a streamlined proteomics-bioinformatics strategy to infer functional interactions from significant similarities in subcellular localisation patterns. COLA uses complete subcellular fractionation in conjugation with quantitative proteomics to generate a quantitative, multi-dimensional, subcellular localisation signature for each identified cellular protein. Bootstrapped hierarchical clustering is then used to match proteins with significant similarity in their localisation signatures. Crucially, COLA is not limited to soluble protein complexes, and can reveal functional interactions on a global scale based on subcellular proximity with high Precision and Sensitivity. Finally, by utilising TandemMass-Tagging for quantitative profiling of different subcellular fractions, we developed a multiplexed version of COLA, named iCOLA, that could be utilised to rapidly map interactomes across different conditions and treatments, thereby revealing interactome dynamics.

\section{Experimental}

\section{Cell-lines, tissue culture, and reagents}

A375P, A375M2, and unlabelled RPE cells were grown in DMEM supplemented with 10\% FBS. For SILAC labelling, RPE cells were grown for at least 7 doublings in SILAC light (plus L-Arg \& L-Lys) or heavy (plus L-Arg10 \& L-Lys8) DMEM supplemented with L-Pro (600 $\mathrm{mg} \mathrm{L}^{-1}$ ) and $10 \%$ dialysed FBS. Rabbit polyclonal antibody against FN1 (sc-9068) was from Santa Cruz. Rabbit monoclonal or polyclonal antibodies against VASP (3132), H2AX (7631), CDH2 (4061), PDI (3501), ERM (3142), and VIM (5174) were from Cell Signalling Technology. Mouse monoclonal antiGAPDH antibody was from Novus Biologicals. Mouse polyclonal antibody against DIS3 (ab-68570) was from Abcam. Mouse monoclonal anti beta-actin (A1978) and anti-EEA1 antibodies were from SIGMA.

\section{Cell lysis and subcellular fractionations (COLA)}

Several types of subcellular fractionation procedures were tested initially; of those four protocols were chosen as they were the most reproducible while providing the most non-overlapping information. These were serial solubilisation, serial centrifugation in combination with aqueous biphasic extraction, transwell protrusion purification, and conditioned media collection (secreted/extracellular fraction). As a whole cell lysate control, an additional dish seeded in the same way was directly lysed by $2 \%$ SDS, Tris-pH 7.6 lysis buffer which solubilises all cellular proteins. For all fractionations, cells were seeded $\left(10^{7}\right.$ per dish $)$ the day before and fractionated in parallel as described below:

(1) Serial solubilisation. For serial solubilisation, Pierce subcellular fractionation kit (78 840) was used with modification. Briefly, cells were scraped off $(1 \times 15 \mathrm{~cm}$ dish $)$ in cold PBS and pelleted before being solubilised serially into 5 fractions according to the kits' protocol (cytosol, membrane, nuclear soluble, nuclear chromatin, and cytoskeleton). A pellet remaining at the end of the procedure was also solubilised by $2 \%$ SDS, Tris-pH 7.6, which constituted a second cytoskeleton fraction.

(2) Serial centrifugation. For serial centrifugation, Abcam plasma membrane extraction kit (ab65400) was used with modification. Briefly, cells were scraped off $(2 \times 15 \mathrm{~cm} \mathrm{dish})$ in cold PBS and pelleted before being homogenised with a Dounce homogenizer in the Homogenize Buffer Mix, according to the kit's protocol. Subsequently, the nuclear fraction (nucleus + envelope) was pelleted by spinning the homogenate at $700 \times g$ for 10 minutes $\left(4{ }^{\circ} \mathrm{C}\right)$, and solubilised in $2 \%$ SDS, Tris-pH 7.6. The supernatant was then further centrifuged at $10000 \times g$ for $30 \mathrm{~min}\left(4^{\circ} \mathrm{C}\right)$ to pellet the cellular membranes. The remaining supernatant (cytosol + microsomes) was taken away, and the pellet was resuspended in $200 \mu \mathrm{l}$ of the 'Upper Phase' aqueous biphasic extraction solution, according to the kit's protocol. An equal volume of 'Lower Phase' solution $(200 \mu \mathrm{l})$ was then added to the mix, vortexed thoroughly, and incubated on ice for 5 minutes, before being centrifuged at $1000 \times g$ for 5 minutes to separate the two phases. In parallel, a fresh tube of mixed upper and lower phase solutions without any sample was similarly prepared and centrifuged to separate the two phase solutions. The upper phase of the tube with samples was then carefully taken away from the lower phase and put in a new tube. The two phases were then extracted again by adding $100 \mu \mathrm{l}$ of the separated lower or upper phase solutions from the tube without samples to each (lower to upper and vice versa) as before (mixing thoroughly, incubating on ice for 5 minutes, before centrifugation at $1000 \times g$ for 5 minutes). The second separated upper phase from the initial upper phase (plasma membrane), and the second separated lower phase from the initial lower phase (intracellular membranes), were then moved to new tubes, diluted in $5 \times$ volume of ice-cold water, and kept on ice for 5 minutes to precipitate the extracted proteins. The proteins were then pelleted by centrifugation at $16000 \mathrm{~g}$ in a microcentrifuge for 10 minutes $\left(4^{\circ} \mathrm{C}\right)$. The supernatants were then removed and discarded and the pellets (intracellular or plasma membrane fractions) were solubilised in 2\% SDS, Tris-pH 7.6. Next, protein concentrations of the fractions were measured by BCA assay (Pierce), and balanced.

(3) Protrusion purification. Cell protrusions were fractionated using $3 \mu \mathrm{m}$ pore polycarbonate transwell filters (corning $75 \mathrm{~mm}$ membrane inserts) as described before. ${ }^{15}$ One transwell per condition was used $\left(10^{7}\right.$ cells per transwell), and cells were allowed to form protrusions through the pores for $4 \mathrm{~h}$. Transwells were then washed in PBS, fixed with $-20{ }^{\circ} \mathrm{C}$ methanol for 20 minutes, washed again with PBS, and the protrusions from the bottom of the filter were shaved off using a glass cover slip, with the cover slip being dipped in 2\% SDS, Tris-pH 7.6 lysis buffer during shaving. The reciprocal SILAC control whole cell lysates were generated by lysing the cells on the top of the filter (cell-bodies) by direct addition of $2 \%$ SDS, Tris-pH 7.6 lysis buffer to the top. Protein concentrations for both protrusions and the cell-bodies were then measured by BCA assay (Pierce), followed by balancing. 
(4) Conditioned media collection. For separation of the extracellular fraction, $10^{7}$ cells were seeded the day before on a $15 \mathrm{~cm}$ dish (1 dish per condition). The next day, media was changed to serum free, and cells were left to secrete proteins overnight into the media, before collecting the media. This conditioned media was subsequently spun at $16000 \mathrm{~g}$ for $10 \mathrm{~min}$ to clear any cell debris, followed by concentrating $\sim 20$ fold using Amicon ultra centrifugal filter units (10 kDa cut-off), and solubilising the concentrated proteins in $2 \%$ SDS, Tris-pH 7.6. As a whole cell lysate control, the remaining cells after removal of the conditioned media were directly lysed by $2 \%$ SDS, Tris-pH 7.6. Again, protein concentrations for both extracellular fraction and the whole cell lysates were measured by BCA assay (Pierce), followed by balancing.

\section{Mass spectrometry sample preparation and LC-MS analysis (COLA)}

Fraction-lysate mixes were trypsinised using the FASP protocol. ${ }^{16}$ Following digestion, peptides were purified by zip-tip C18 clean-up tips (Millipore), lyophilised using a speedvac, and the dried peptides were then reconstituted in $1 \%$ acetonitrile/ $0.1 \%$ formic acid for LC-MS/MS. LC-MS/MS runs were performed by ICR's proteomics core facility as described before, ${ }^{15}$ with minor modifications. Briefly, reversed phase chromatography was performed using an HP1200 platform (Agilent, Wokingham, UK). One third of each sample was analysed as a $6 \mu$ injection. Peptides were resolved on a $75 \mu \mathrm{m}$ I.D. $15 \mathrm{~cm}$ C18 packed emitter column ( $3 \mu \mathrm{m}$ particle size; Nikkyo Technos Co., Ltd, Tokyo, Japan) over 240 min using a three-step gradient of $96: 4$ to $50: 50$ buffer $\mathrm{A}: \mathrm{B}(t=0 \min 4 \% \mathrm{~B}, 0.5 \min 4 \% \mathrm{~B}$, $40.0 \min 10 \%$ B, 170.0 min $25 \%$ B, 240.0 min $50 \%$ B) (buffer A: $1 \%$ acetonitrile $/ 3 \%$ dimethyl sulfoxide $/ 0.1 \%$ formic acid; buffer B: $80 \%$ acetonitrile/3\% dimethyl sulfoxide $/ 0.1 \%$ formic acid) at $250 \mathrm{~nL} \min ^{-1}$. Peptides were ionised by electrospray ionisation using $1.8 \mathrm{kV}$ applied immediately precolumn via a microtee built into the nanospray source. Sample was injected into an LTQ Velos Orbitrap mass spectrometer (Thermo Fisher Scientific, Hemel Hempstead, UK) directly from the end of the tapered tip silica column (6-8 $\mu \mathrm{m}$ exit bore). The ion transfer tube was heated to $275{ }^{\circ} \mathrm{C}$ and the S-lens set to $60 \%$. MS/MS were acquired using data dependent acquisition based on a full 30000 resolution FT-MS scan with preview mode disabled. The top 20 most intense ions were fragmented by collision-induced dissociation and analysed using normal ion trap scans. Precursor ions with unknown or single charge states were excluded from selection. Automatic gain control was set to 1000000 for FT-MS and 30000 for IT-MS/MS, full FT-MS maximum inject time was $500 \mathrm{~ms}$ and normalised collision energy was set to $35 \%$ with an activation time of $10 \mathrm{~ms}$. Wideband activation was used to co-fragment precursor ions undergoing neutral loss of up to $-20 \mathrm{~m} / \mathrm{z}$ from the parent ion, including loss of water/ammonia. MS/MS was acquired for selected precursor ions with a single repeat count acquired after $8 \mathrm{~s}$ delay followed by dynamic exclusion with a $10 \mathrm{ppm}$ mass window for $60 \mathrm{~s}$ based on a maximal exclusion list of 500 entries.

\section{Mass spectrometry sample preparation and LC-MS analysis (iCOLA)}

For iCOLA, $100 \mu \mathrm{g}$ of each subcellular fraction (Fig. 3A) was digested by FASP, amine-TMT-10-plex labelled (Pierce 90111) on filter membranes (iFASP), ${ }^{17}$ eluted, pooled, and lyophilised. Peptides were desalted using C18 solid-phase extraction (SPE). LC-MS3 analysis of TMT labelled peptides was performed by Cancer Research UK Manchester Institute's proteomics core facility. Briefly, reverse-phase chromatographic separation was performed on an RSLCnano (Thermo Scientific) with a PepMap RSLC C18 $(2 \mu \mathrm{m}$ bead size $), 100$ A, $75 \mu \mathrm{m}$ I.D. $\times 50 \mathrm{~cm}$ EasySpray unit at $60 \mathrm{C}$ using a $120 \mathrm{~min}$ linear gradient of $0-50 \%$ solvent B (MeCN $100 \%+0.1 \%$ formic acid (FA)) against solvent $\mathrm{A}\left(\mathrm{H}_{2} \mathrm{O} 100 \%+0.1 \% \mathrm{FA}\right)$ with a flow rate of $300 \mathrm{~nL} \mathrm{\textrm {min } ^ { - 1 }}$. The separated samples were infused into an Orbitrap Fusion mass spectrometer (Thermo Scientific). The mass spectrometer was operated in the data-dependent mode to automatically switch between Orbitrap MS and MS/MS acquisition. Survey full scan MS spectra (from $\mathrm{m} / \mathrm{z} 300-2000$ ) were acquired in the Orbitrap with a resolution of 120000 at $\mathrm{m} / \mathrm{z} 400$ and FT target value of $1 \times 106$ ions. The 20 most abundant ions were selected for MS2 fragmentation (isolation window $1.2 \mathrm{~m} / \mathrm{z}$ ) using collisioninduced dissociation (CID), dynamically excluded for 30 seconds, and scanned in the ion trap at 30000 at $\mathrm{m} / \mathrm{z} 400$. MS3 multi-notch isolated ions (10 notches) ${ }^{18}$ were fragmented using higher-energy collisional dissociation (HCD) and scanned in the Orbitrap (from $\mathrm{m} / \mathrm{z} 100-500)$ at 60000 at $\mathrm{m} / \mathrm{z} 400$. For accurate mass measurement, the lock mass option was enabled using the polydimethylcyclosiloxane ion $(\mathrm{m} / \mathrm{z} 445.12003)$ as an internal calibrant. Four serial technical replicate injections were performed per TMT sample set to boost the identification coverage.

\section{Proteomics search and quantifications}

Mass-spectrometry search and SILAC/TMT quantifications were performed by Maxquant. ${ }^{19}$ The search was performed against the Human Uniprot database, with a false detection rate (FDR) of $1 \%$ for both peptides and protein identifications, calculated using reverse database search. Second-peptide search, match between runs (using a 2 minutes matching window), and re-quantify options were all enabled to achieve maximum quantification depth. Only razor or unique, unmodified peptides, as well as methionine oxidized peptides were used for quantification. To achieve higher coverage and better matching of SILAC samples, all raw files were searched together. Following the search, preliminary data analysis on the search results was performed by Perseus software from the maxquant package. ${ }^{20}$ Briefly, reverse, contaminants, and proteins identified from only modified peptides were filtered out. For iCOLA, all fraction reporter ion channels were normalised to the total lysate channel. Ratios were transformed to $\log 2$ scale. For all further downstream co-localisation analysis, data from each replicate (reciprocally labelled SILAC replicates or multiple injection TMT replicates) was averaged and $z$-scored, generating a single value per protein per fraction for each experiment. Proteins were filtered to have ratio values for all fractionations. $2 \mathrm{D}$ annotation 
enrichment analysis was performed by Perseus software as described in ref. 21, using GO, GSEA, CORUM, Pfam, SMART, KEGG, and Uniprot Keyword annotations. A Benjamini-Hochberg false detection rate of $2 \%$, and an enrichment cut off delta score of +0.2 per category was applied. PCA analysis was also performed by Perseus using the averaged $z$-scored values. Pearson correlation analysis was also performed by Perseus prior to averaging.

\section{Co-localisation identification}

Interactions between proteins were defined as proteins that their localisation patterns significantly and robustly cluster together in different bootstrapped samples. It was performed using pvclust function in $R$ with Euclidean distance, average linkage, and AU (Approximately Unbiased) $p$-value measure ${ }^{22}$ (https://cran.r-project.org/web/packages/pvclust/). 500 (for iCOLA) or 1000 bootstrappings were performed and only clusters with $p$-value $<0.05,0.01$, or 0.001 were considered. If a cluster consisted of more than 2 proteins, then all possible pairwise interactions were considered.

\section{Interaction overlap analysis}

Interactions that were identified using bootstrapped clustering were evaluated by calculating the overlap with the following protein-protein interaction reference databases:

(1) STRING-all interactions: ${ }^{23}$ all string interactions were downloaded (Oct 2014). String gene IDs were mapped to corresponding gene IDs using UNIPROT ID mapping tool. Interactions with medium confidence (combined score $>0.4$ ) were considered where the score is based on neighbourhood, gene fusion, co-occurrence, co-expression, experiments (physical interactions), databases and text mining methods.

(2) STRING-physical interactions: ${ }^{23}$ interactions that have experimental evidence in STRING.

(3) Pathway Commons: ${ }^{24}$ based on Pathway Commons 7 (May 2015) with exclusion of BioGrid as this database includes the studies we used for benchmarking our methods against (see below).

(4) CORUM $^{25}$ (protein complex database): gene IDs were mapped using UNIPROT.

The overlap was calculated as the percentage of identified interactions in COLA or ICOLA that were also reported in the above mentioned databases. The significance of the overlap was calculated using right tail Fisher Exact Test $(R)$ and hypergeometric probability. For the number of interactions in the reference databases, only interactions that include the proteins that were quantified in our fractionation experiments were considered. We benchmarked our method against Kristensen et al., ${ }^{12}$ (7204 interactions), and Rolland et al., ${ }^{6}$ (13944 interactions). To calculate the significance of overlap, the number of interactions in reference database was modified to only include the proteins that were identified using each of these methods.

\section{Mitochondrial flux analysis}

Mitochondrial flux analysis was performed as described before. ${ }^{26}$ Briefly, A375P and A375M2 cells were plated at $2 \times 10^{4}$ cells per well of a XF Mito Stress Kit plate (Agilent) $(n=18)$ and oxygen consumption was measured in real-time using a XFe 96 Analyzer (Seahorse Biosciences) for 70 minutes. $1 \mu \mathrm{M}$ oligomycin, $0.25 \mu \mathrm{M}$ FCCP, and $0.5 \mu \mathrm{M}$ rotenone/antimycin (R/A) were serially added at indicated time-points to assess basal mitochondrial, maximal mitochondrial, and non-mitochondrial oxygen consumption rates, respectively. Cell numbers were normalised using Cyquant Kit (Thermo Fisher).

\section{Interactome quality assessment}

COLA and ICOLA (A375M2) derived binary interactions, as well as interactions of Kristensen et al., ${ }^{12}$ and Rolland et al., ${ }^{6}$ were evaluated against STRING, Pathway Commons, and CORUM databases. To measure Sensitivity, the percentage of CORUM interactions that were identified in each method, from the total number of CORUM interactions for the observed proteins was calculated. For measurement of False Positive Rate (FPR), we employed two different approaches. In the first approach, we first generated a list of all hypothetical binary interactions (based on identified proteins for each method). All known interactions that were reported in STRING, Pathway Commons, and CORUM were then filtered out. Next, we took 1000 random samples of interactions from the remaining interactome. Sample size was set to 200 interactions. ${ }^{27}$ FPR for each method was then calculated by finding the percentage of the 200 interactions that were reported each time. The values were then averaged and reported. In the second approach, we generated a list of all possible hypothetical binary interactions based on the list of the identified proteins in each method that have a CORUM annotation. All known binary interactions that were reported in STRING, Pathway Commons, and CORUM were then filtered out. This list was termed anti-CORUM. FPR was then calculated by finding the percentage of anti-CORUM interactions that were reported by each method. To estimate Precision, we used Sensitivity, and anti-CORUM based FPR values to estimate the number of true positives (S), false positives (V), true negatives (U), and false negatives (T) for each method, as defined in (Table 3). Precision was then calculated as $\mathrm{S} /(\mathrm{S}+\mathrm{V})$.

\section{Results}

\section{Analysis of functional interactions by COLA}

To generate quantitative subcellular localisation signatures for analysis of colocalisation, we first developed a multi-variate method to assess subcellular localisation of proteins by proteomics. We used Stable Isotope Labelling of Amino acids in Culture (SILAC) in conjugation with extensive subcellular fractionation ${ }^{28}$ (Fig. 1A). SILAC experiments were carried out in duplicate with label switching, and an average ratio was calculated for each protein per each fraction, using whole cell lysate as standard (Fig. 1A). Protein localisation signatures derived from combining all fractions were then clustered using bootstrapped hierarchical clustering to reveal similar signatures with high confidence (Fig. 1A).

To maximise acquisition of novel, digitised, information on subcellular protein distributions which would be suitable for 

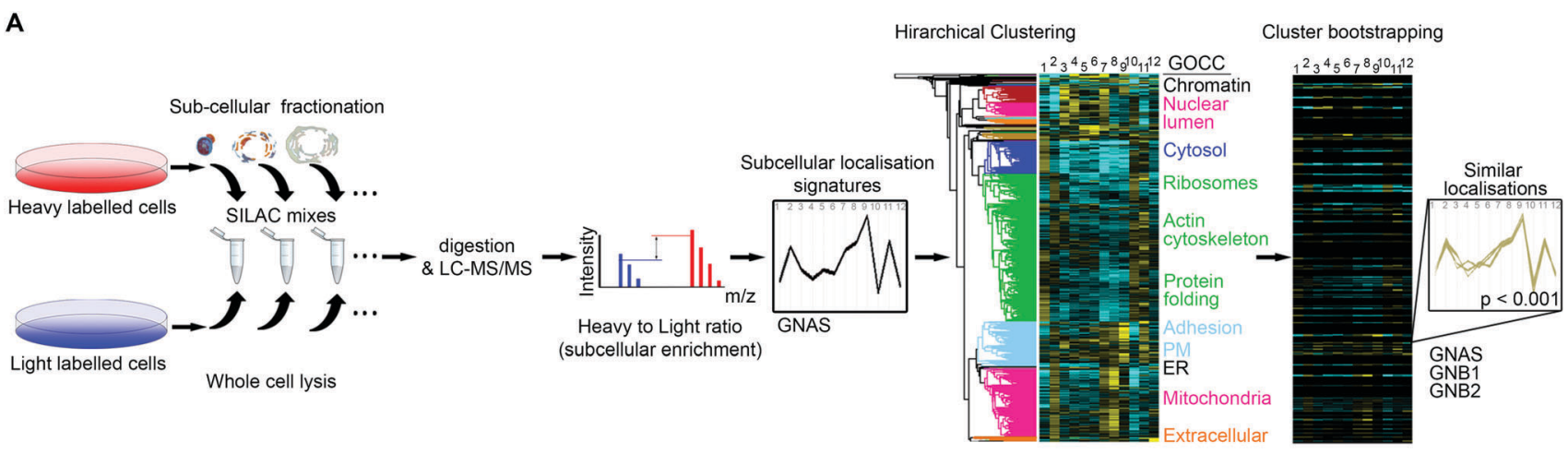

B

C

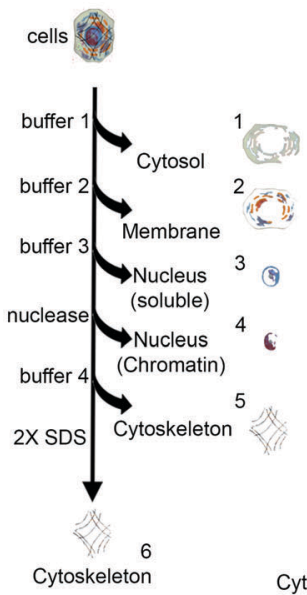

Cytosol (+microsomes)
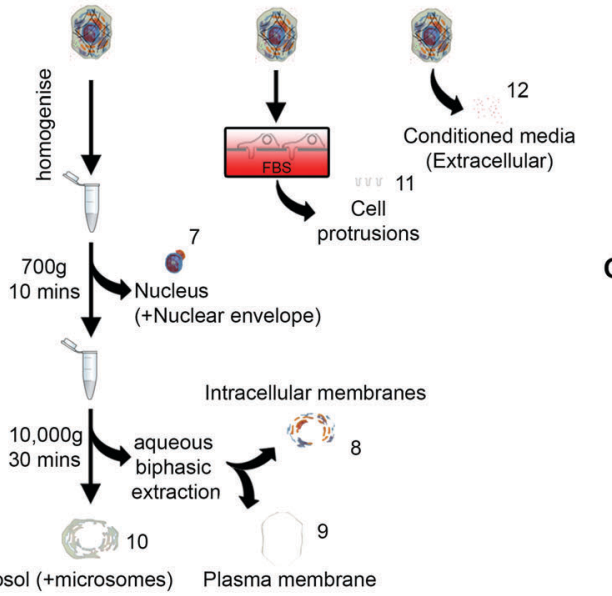
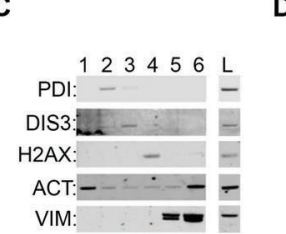

G

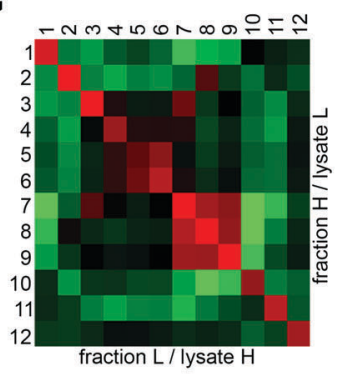

E
I

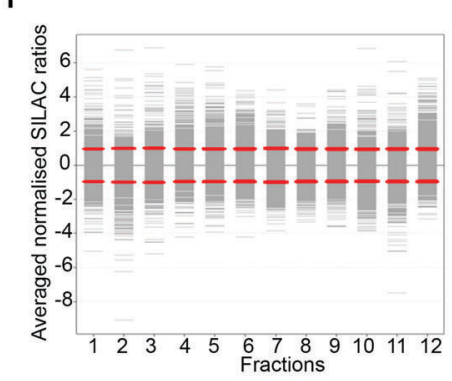

K

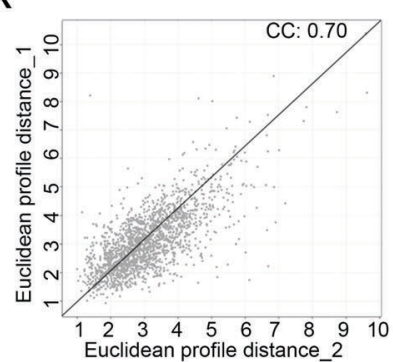

J

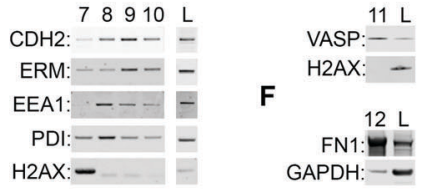

H

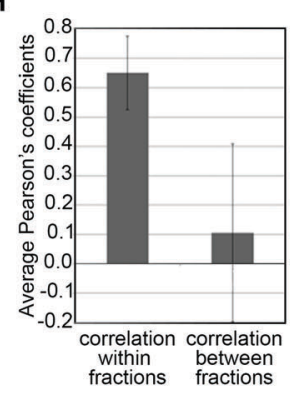

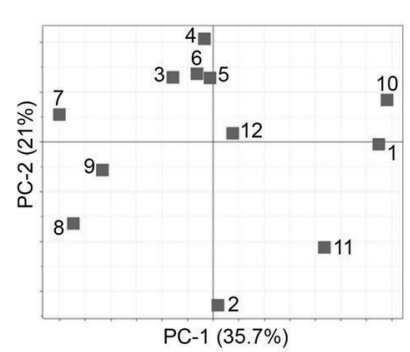

L

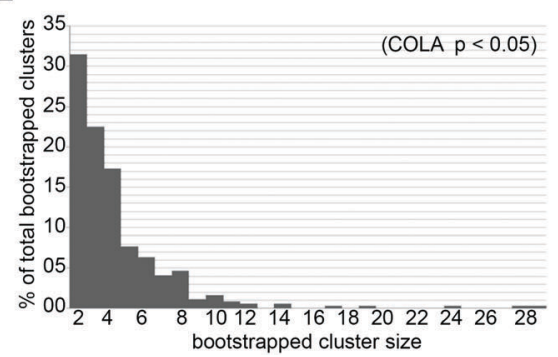

Fig. 1 Proteomics-based profiling of colocalisation (COLA) to reveal functional associations. (A) Outline of methodology. SILAC heavy or light labelled cells were subjected to subcellular fractionation then mixed with an equal amount of whole cell lysate from the opposite label for relative quantification. SILAC mixes were digested and analysed by LC-MS/MS. Averaged normalised SILAC ratios for all fractions together create a multi-variate localisation signature for each protein (GNAS was used as example). Signatures were then subjected to unsupervised hierarchical clustering with Euclidean average linkage. Bootstrapping was used to reveal clustering matches with high confidence (in color) from the rest (blacked out). GNAS, GNB1, and GNB2 which are known to constitute a complex are shown as example of a bootstrapped cluster. (B) Outline of the subcellular fractionations. Four independent fractionations procedures were used: (1) serial solubilisation of cellular proteins (fractions 1 to 6), (2) serial centrifugation combined with aqueous biphasic extraction which separates plasma membrane from internal membranes (fractions 7 to 10), (3) separation of actin-rich cellular protrusions using microporous transwell filters (fraction 11), (4) separation of the extracellular/secreted proteins by collecting conditioned media (fraction 12). (C) Validation of serial solubilisation method (fractions 1-6) by western blotting: PDI, ER membrane protein; DIS3, soluble nuclear protein; H2AX, nucleosome constituent; 
actin (ACT) and vimentin (VIM), cytoskeletal proteins; $L$ = matching whole cell lysate control. (D) Validation of serial centrifugation method (fractions 7-10) by western blotting: $\mathrm{N}$-cadherin (CHD2) and ezrin/radixin/moesin proteins (ERM), plasma membrane; early endosomal antigen-1 (EEA1), endosomal; PDI, ER membrane protein; $\mathrm{H} 2 \mathrm{AX}$, nucleosome constituent; $\mathrm{L}=$ matching whole cell lysate control. (E) Validation of protrusion purification method (fraction 11) by western blotting: VASP, protrusion; H2AX, nuclear; $L=$ matching cell-body lysate control. (F) Validation of conditioned media collection method (fraction 12) by western blotting: fibronectin-1 (FN1), secreted; GAPDH, intracellular; $L$ = matching whole cell lysate control. (G) Heat map of Pearson correlation coefficients between the two SILAC replicate series of fractionations with switched labelling. Cells were fractionated in duplicate with switching the labels. Collected fraction mixes have high similarity with their corresponding replicate, but low similarity with other fractions. (H) Plotted averaged Pearson's correlation coefficients within replicate fractions versus averaged Pearson's correlation coefficients between different fractions. While a high degree of similarity exists within replicate fractions suggestive of high reproducibility, similarity between different fractions is very low, indicating that each fraction is likely providing unique information. (I) Distribution of SILAC ratios for each fraction (averaged from two replicates). Red lines mark the standard deviations for each fraction. (J) Principle component analysis of subcellular fractions. PC1 and 2 represent over $50 \%$ of all data variation. No single fraction contributes towards the overall variation disproportionately. (K) Analysis of the reproducibility of the overall fractionation signatures between two biological replicates. Euclidean distances between each protein signatures from two reciprocally SILAC labelled COLA fractionations were calculated and plotted against each other, showing a highly significant correlation $\left(p<1.0 \times 10^{-15}\right)$. The Pearson correlation coefficient (CC) is displayed on the graph. (L) Graph of percentage of total bootstrapped clusters $(p<0.05)$ vs. the number of proteins per cluster. The majority of clusters are constituted of 2-4 proteins, yet very large clusters are still detectable by COLA.

hierarchical clustering, we used four parallel independent fractionation procedures with highly distinct individual fractions (Fig. 1B). The majority of subcellular fractions came from two main fractionation procedures, one based on serial solubilisation by using successive solubilising buffers, and the other based on serial centrifugation (Fig. 1B). Both of these procedures are fast, reproducible, require little optimisation, and cover major subcellular compartments. To further expand on subcellular information, we also collected two additional independent fractions, the actin-rich cellular protrusions, and the extracellular compartment (Fig. 1B). Protrusions are purified using transwell based physical separation of cell protrusions from the cell-body, ${ }^{15}$ and the extracellular compartment is collected by removing and concentrating conditioned media (Fig. 1B). Overall, we identified 4950 proteins from human Retinal Pigment Epithelial (RPE) cells, out of which 1886 had a full subcellular localisation profile with no missing values (Dataset S1, ESI $\dagger$ ). The quality of fractionations was examined by western blotting for markers of specific fractions (Fig. 1C-F), as well as category enrichment analysis using the Gene Ontology Cellular Compartment (GOCC) database (Table 1 and Dataset S2, ESI $\dagger$ ). For assessing the reproducibility of fractionations, we determined Pearson's correlation coefficients across all fraction replicates. While correlation coefficient within fraction replicates was on average 0.65 , suggestive of good reproducibility of fractionations, coefficients between different fractions was only 0.1 on average (Fig. 1G and H), indicating that fractions provide unique information towards the localisation signatures. The distribution and variance of different fractions were comparable (Fig. 1I), and importantly, no single fraction contributed disproportionately to the overall variance (Fig. 1J), ruling out potential bias towards a specific compartment in later downstream analysis. The correlation between the overall subcellular localisation signatures, across two independent biological replicates was calculated to be 0.70 (Fig. 1K).

To detect proteins that have significantly similar localisation signatures, and therefore are expected to functionally interact, we used hierarchical clustering (Fig. 1A). Hierarchical clustering is ideal for multi-variate assessment of functional interactions as it matches proteins into discrete functional units. ${ }^{3}$ However, a common shortcoming of standard hierarchical clustering is sensitivity to samples order and variation in clustering results depending on sample inclusion. ${ }^{22}$ To ensure the significance and robustness of our clustering to permutations, we performed bootstrapped clustering to reveal groups of proteins that group together with high confidence, thus are likely to be truly colocalising (Fig. 1A). We used three different bootstrapping stringency cut-offs of $p$-value $<0.05,0.01$, or 0.001 , revealing 365, 271, or 101 bootstrapped clusters (Dataset S3, ESI $\dagger$ ), which correspond to 4415,3087 , or 1487 pair-wise co-localisations, respectively (Dataset S4, ESI $\dagger$ ). While over $50 \%$ of identified bootstrapped clusters contain only 2 or 3 proteins, clusters of 10 or more proteins were also detected (Fig. 1L), suggesting that COLA can detect both small and large complexes.

Table 1 Top GOCC category enrichments in each COLA fractions. Selected highly enriched terms from (Dataset S2, ESI) are listed

\begin{tabular}{ll}
\hline Fraction & Significantly enriched GOCC terms (FDR < 0.02) \\
\hline 1 & Cytosol; intracellular \\
2 & Membrane part; integral component of plasma-membrane; organelle membrane \\
3 & Nuclear part; nucleoplasm part; transcription factor complex; chromatin remodelling complex; chromatin \\
4 & Nuclear part; protein-DNA complex; nucleosome; chromosome; nuclear chromosome part; nuclear body \\
5 & Intermediate filament; nuclear membrane; nucleolus \\
6 & Intermediate filament; nuclear membrane; nucleolus \\
7 & Protein-DNA complex; chromatin remodelling complex; nucleoid; organelle part; membrane part \\
8 & Organelle membrane; membrane enclosed lumen; respiratory chain complex \\
9 & Plasma membrane; cell junction; coated pit; endosome membrane; membrane part; protein-DNA complex \\
10 & Cytosolic small ribosomal subunit; cytosolic large ribosomal subunit; proteosome complex; MCM complex \\
11 & Actin cytoskeleton; ruffle; cell projection; cell junction; adherens junction; synapse; plasma membrane \\
12 & Extracellular space; extracellular matrix; basement membrane; secretory granule lumen
\end{tabular}


A

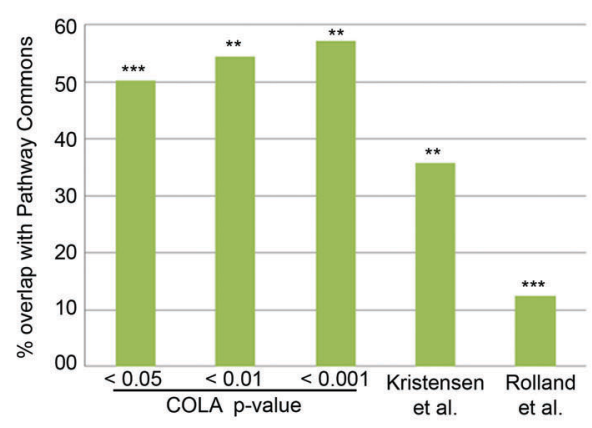

C

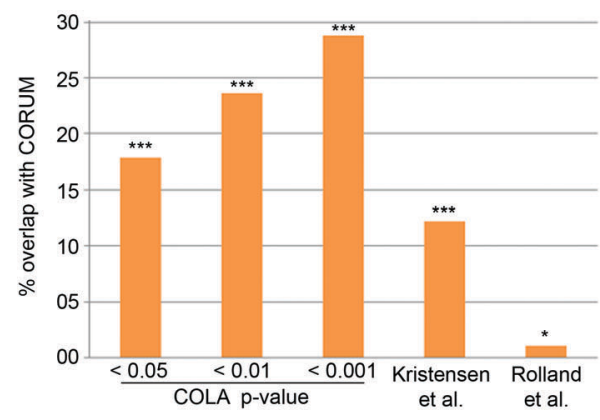

B

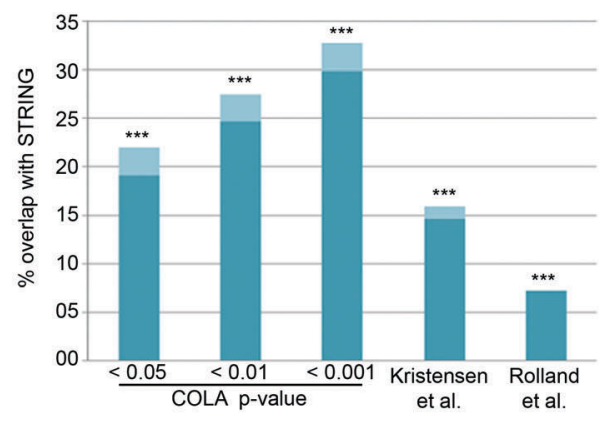

D

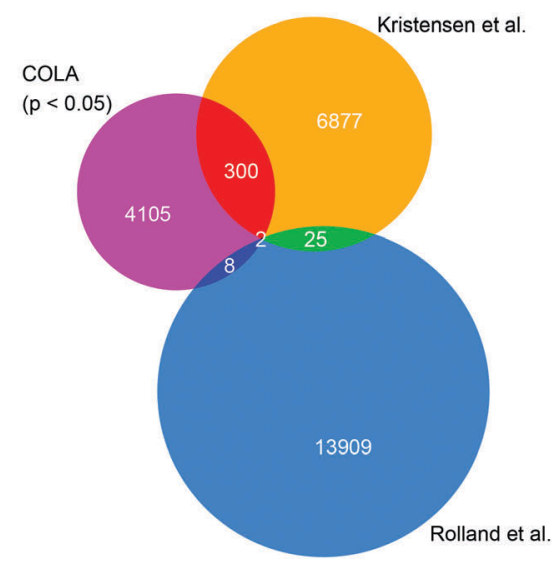

Fig. 2 COLA reveals known functional associations and outperforms two current global interactome analysis studies. (A) Percentage of overlap with Pathway Commons between the identified interactions in each bootstrap significance setting, vs. in Kristensen et al., ${ }^{12}$ vs. in Rolland et al., ${ }^{6} P^{\star * *}$ : fisher's exact test $p<1 \times 10^{-300 ;}$;** fisher's exact test $p<2 \times 10^{-150 ;}$; : fisher's exact test $p<1 \times 10^{-75}$. (B) As in (A) but for overlap with STRING (darker blue indicates physical interactions only). (C) As in (A) but for overlap with CORUM. (D) Venn diagram of the overlap between binary protein-protein interactions revealed COLA versus Kristensen et al., size-exclusion chromatography profiling ${ }^{12}$ and Rolland et al., Y2H screening. ${ }^{6}$ Only 2 interactions are shared across the three methods.

To verify that COLA predicts real interactions, we assessed its performance in detecting known interactions using three major mammalian functional interaction databases as reference: (1) CORUM, a highly curated database of mammalian protein complexes, ${ }^{25}$ (2) STRING, a larger database of both physical and functional associations. ${ }^{23}$ (3) Pathway Commons, a comprehensive collection of functional and physical interactions integrated from multiple publicly available databases. ${ }^{24}$ We quantified the proportion of co-localising proteins in our method that appear as known functional interactors in each database (Dataset S4, $\mathrm{ESI} \dagger$ ). Over than half of the COLA identified interactions are annotated as known interactions based on the Pathway Commons database - the largest collection of functional protein interactions (Fig. 2A). Moreover, around 1 in five of COLA interactions are annotated as known in STRING database (Fig. 2B), and a similar percentage of overlap with CORUM interaction database was also detected (Fig. 2C). All of these degrees of overlap are statistically highly significant ( $p$-value $<1 \times 10^{-300}$ ). For comparison, we also evaluated the performance of two published large-scale studies which used different approaches to map human protein-protein interactions as a proxy for functional associations: (1) Rolland et al., which used $\mathrm{Y} 2 \mathrm{H}$ screening, ${ }^{6}$ and (2) Kristensen et al., which used size-exclusion chromatography coupled with proteomics (SEC-MS). ${ }^{12}$ Although both studies significantly detected known interactions, they were both consistently outperformed by COLA in every database comparison (Fig. 2A-C). Interestingly, the overlap between each of these three approaches was little despite the significant degree of overlap with the reference databases, suggesting that each method must be revealing complementary information with regards to functional associations (Fig. 2D). These results demonstrate that significant similarity in protein localisation signatures is strongly reflective of a functional interaction, and that COLA outperforms Y2H and SEC-MS methods in revealing functional interactions.

\section{Analysis of interactome dynamics by iCOLA}

Next, we optimised COLA for analysis of interactome dynamics. A key factor for revealing the dynamics of protein interaction networks is the ability to reliably multiply interactome analyses across several conditions. Thus, high reproducibility along with short analysis times are two crucial criteria for any method designed for analysis of interactome dynamics. The SILAC based COLA method requires a single mass spectrometry run for every fraction/lysate SILAC mix. This equates to a total of 24 runs $(2 \times 12$ reciprocally labelled fraction/lysate mixes) for every analysis, 
A

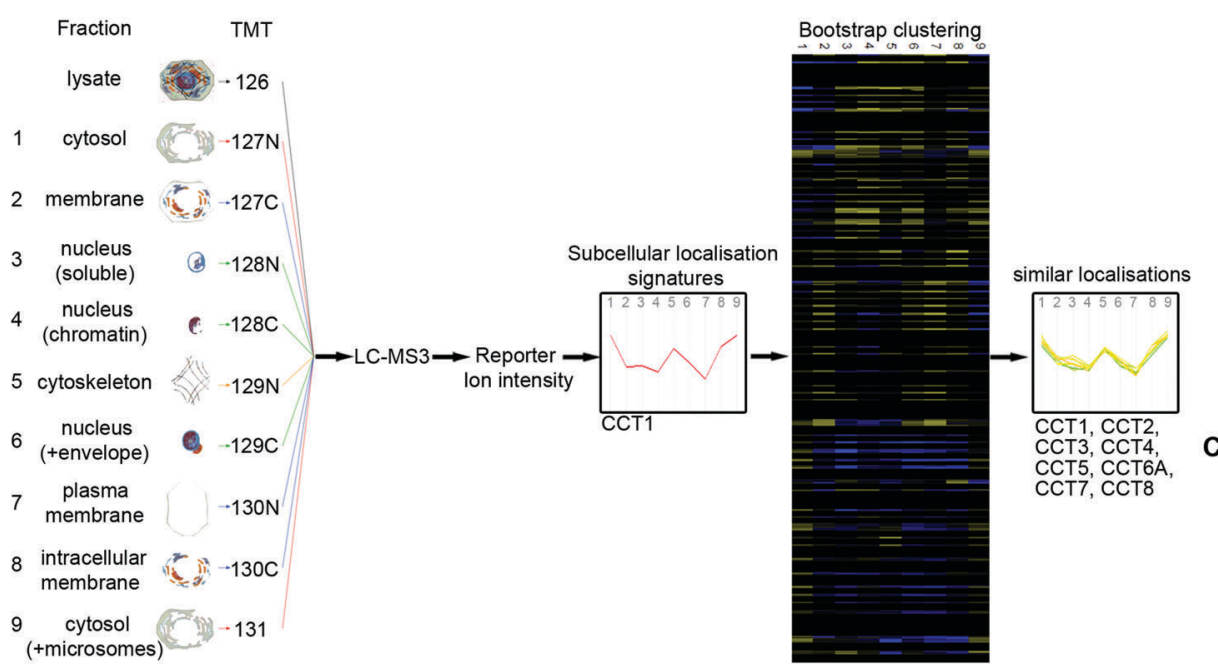

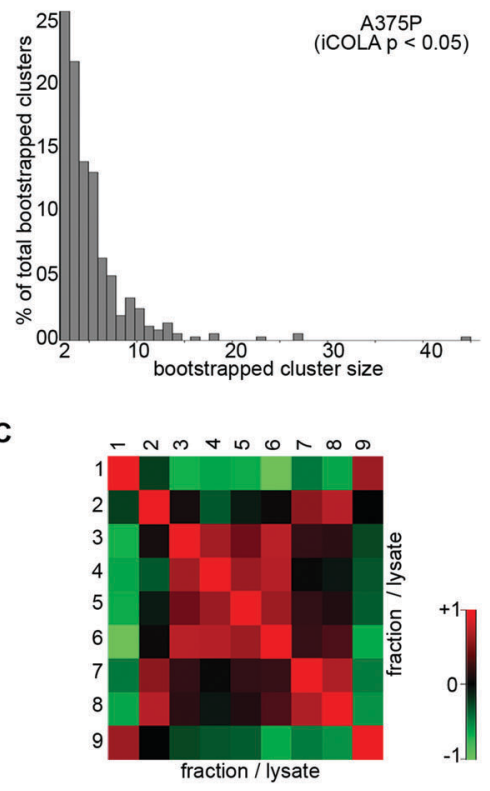

G

H

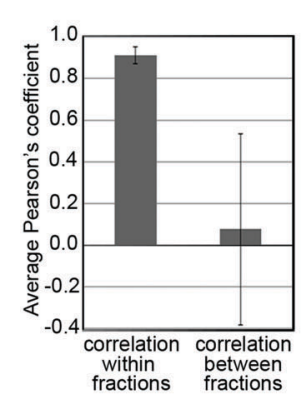

I

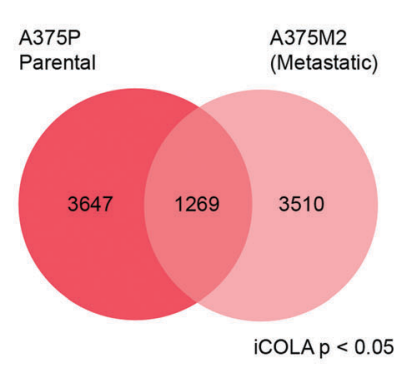

E

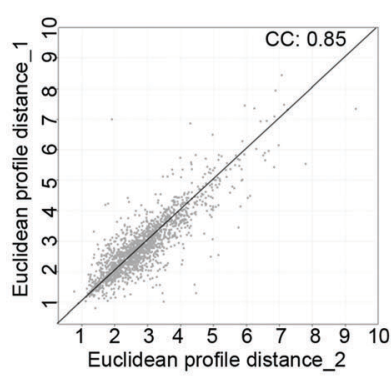

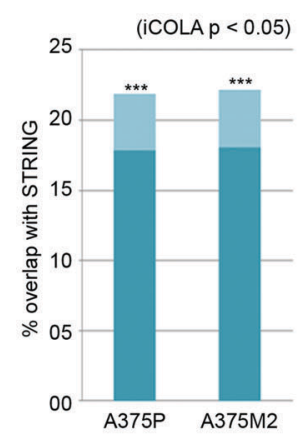

J
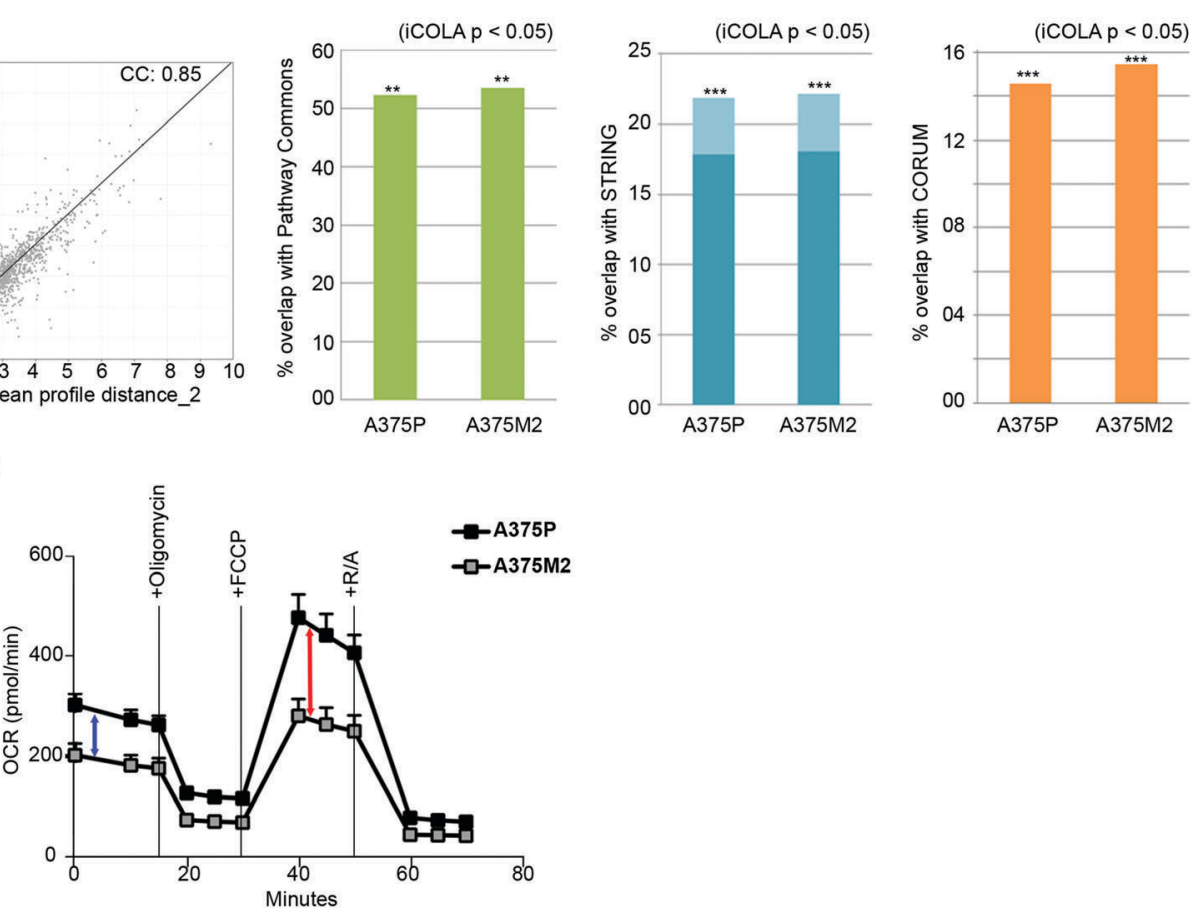

Fig. 3 Analysis of interactome dynamics by iCOLA. (A) Outline of the iCOLA methodology. 9 fractions from serial solubilisation and serial centrifugation protocols, along with a $2 \%$ SDS solubilised whole cell lysate total control, were digested and isobarically labelled using TMT 10-plex kit as indicated. The labelled peptides were then pooled together and analysed by LC-MS3. Averaged normalised fraction/lysate ratios for every fraction were used to create a multi-variate subcellular localisation signature for each protein (T-complex protein 1 subunit alpha was used as example here). Signatures were then subjected to unsupervised hierarchical clustering with Euclidean average linkage and bootstrapping was used to reveal clustering matches with high confidence (in color) from the rest (blacked out). All members of the TCP1 chaperonin ring complex (CCT1 to 8) are detected as significant interactors of CCT1, and are shown as an example of a bootstrapped cluster. (B) Graph of percentage of total bootstrapped clusters ( $p<0.05)$ vs. the number of proteins per cluster from iCOLA analysis of A375P cells. The majority of clusters are constituted of 2-4 proteins, yet very large clusters are still detectable by iCOLA. (C) Heat map of Pearson correlation coefficients between the two replicate iCOLA series of fractionations. Cells were fractionated in duplicate. Collected fractions show high similarity with their corresponding replicate, but low similarity with other fractions. (D) Plotted averaged Pearson's correlation coefficients within replicate fractions versus averaged Pearson's correlation coefficients between different fractions. While a high degree of similarity exists within replicate iCOLA fractions suggestive of high reproducibility, similarity between different fractions is very low, indicating that each fraction is likely providing unique information. (E) Analysis of the reproducibility of the overall localisation signatures between two biological replicate iCOLA experiments. Euclidean distances between the signatures from two independent iCOLA fractionations experiments in A375P cells were calculated 
and plotted against each other, showing a highly significant correlation $\left(p<1.0 \times 10^{-15}\right)$. The Pearson correlation coefficient (CC) is displayed on the graph. (F) Comparison of the percentage of known interactions according to Pathway Commons that were detected in A375P and A375M2 cells $(p<0.05)$. Percentage of overlap with Pathway Commons was very similar in A375P and A375M2 cells, and comparable to SILAC based COLA (Fig. 2A). $\star \star \star$ : fisher's exact test $p<1 \times 10^{-300}$; **: fisher's exact test $p<2 \times 10^{-200}$. (G) Comparison of the percentage of known interactions according to STRING that were detected in A375P and A375M2 cells $(p<0.05)$. Light blue bars shows all STRING interactions. Dark blue bars show only physical interactions. Percentage of overlap with STRING was very similar in A375P and A375M2 cells, and comparable to SILAC based COLA (Fig. 2B). ***: fisher's exact test $p<1 \times 10^{-300} ; * *$ : fisher's exact test $p<2 \times 10^{-200}$. (H) Comparison of the percentage of known interactions according to CORUM that were detected in A375P and A375M2 cells ( $p<0.05$ ). Percentage of overlap with CORUM was very similar in A375P and A375M2 cells, and comparable to SILAC based COLA (Fig. 2C). ***: fisher's exact test $p<1 \times 10^{-300} ; * *$ : fisher's exact test $p<2 \times 10^{-200}$. (I) Venn diagram of the overlap between binary protein-protein interactions detected in A375P and A375M2 cells (bootstrap cut off $=0.05$ ). A core of 1269 interactions were conserved between the two isogenic cell lines, while over 3000 unique interactions were detected in each cell-line. (J) Analysis of mitochondrial respiratory flux in A375P and A375M2 cells. Oxygen consumption (OCR) was measured in real-time, with serial addition of oligomycin to inhibit ATP synthase, FCCP to uncouple oxygen consumption from ATP production, and rotenone/antimycin (R/A) to completely inhibit electron transport chain, at indicated timepoints. Values were normalised to total seeded cell numbers. A375P show a significantly higher basal mitochondrial respiration (blue arrow), as well as a higher maximal mitochondrial respiratory capacity (red arrow), while levels of non-mitochondrial oxygen consumption measured after R/A addition (three ending time points) are equal between both cells.

which translates into a total mass spectrometry run time of around 5 days, using our LC-MS/MS settings. To reduce the number of mass spectrometry runs required in order to allow rapid analysis of interactomes across multiple conditions, we developed a modified version of our method (iCOLA), utilising Tandem Mass Tagging (TMT) isobaric labelling which allows mixing and quantification of up to 10 different samples in a single mass spectrometry run. ${ }^{29}$ To have all the fractions analysed in a single TMT run, we modified our fractionation procedure to have a total of 9 fractions plus 1 whole cell lysate (Fig. 3A). We focused on serial solubilisation and serial centrifugation procedures which provide the bulk of subcellular coverage, and also mixed the two cytoskeletal fractions from serial solubilisation protocol, which had the highest degree of similarity (Fig. 1J), into one fraction (Fig. 3A). Co-localisations were identified as before by determining significant similarities between subcellular localisation signatures using bootstrapped clustering (Fig. 3A). We applied iCOLA to analyse co-localisations in A375P melanoma cells. $^{30}$ Overall, we identified 2276 proteins, complete subcellular localisation profiles of 1846 of which were defined across all fractions in A375P cells (Dataset S5, ESI $\dagger$ ). At the bootstrap cut-off of $p$-value $<0.05$, a total of 357 bootstrap clusters were revealed (Dataset S6, ESI $\dagger$ ), corresponding to 4916 pair-wise co-localisations (Dataset S7, ESI $\dagger$ ). Similar to the SILAC based COLA, the majority of iCOLA identified bootstrapped clusters contained only 2 or 3 proteins, but clusters of 10 or more proteins were also detected (Fig. 3B), suggesting that iCOLA performs comparably to the SILAC based COLA in detecting both small and large complexes. To assess iCOLA's reproducibility, we determined Pearson's correlation coefficients across all fraction replicates as before. Correlation coefficient within fraction replicates was on average 0.92 , which is better than COLA, while coefficients between different fractions was on average 0.08 (Fig. 3C and D). In addition, the correlation between the overall fractionation signatures across two independent biological replicates was calculated to be 0.85 (Fig. 3E), which is also better than that of COLA. The improved reproducibility of iCOLA is likely due to the fact that protein quantifications are derived from the same peptides in every fraction, as every identified TMT labelled peptide returns a quantification value for each fraction. In contrast, the same protein can be quantified through different SILAC labelled peptides in each fraction/lysate mix of COLA, thus increasing the likelihood of noise.

Next, we applied iCOLA to reveal interactome differences between our previously analysed A375P cells, which are weakly metastatic, to their highly metastatic isogenic derivative, the A375M2 cells. $^{30}$ We identified 1442 proteins with complete subcellular localisation profiles in A375M2 cells (Dataset S8, ESI $\dagger$ ). At the bootstrap cut-off of $p$-value $<0.05$, a total of 279 bootstrap clusters were revealed for A375M2 cells, (Dataset S9, $\mathrm{ESI} \dagger$ ), corresponding to 4779 pair-wise co-localisations (Dataset S10, $\mathrm{ESI} \dagger)$. First, to test whether the performance of iCOLA is similar to COLA in terms of identifying true functional interactions in both cell-types, we assessed the overlap of the identified co-localisations with CORUM, STRING, and Pathway Commons interaction databases, as before (Datasets S7 and S10, ESI $\dagger$ ). A highly significant proportion of the revealed co-localisations were amongst known functional interactors in both A375P and A375M2 cells (Fig. 3F-H), with the degree of overlap being comparable to that of the SILAC based COLA method, suggesting that reducing the number of fractions from 12 to 9 does not significantly affected the ability of our approach to reveal true interactions. Next, we assessed the degree of overlap between the interactomes of the two cell-lines. 1269 of the interactions identified in total were seen in both A375P and A375M2 cells, whilst more than 3000 interactions were detected in only one cell-type (Fig. 3I). Category enrichment analysis revealed that most conserved interactions belonged to core cellular complexes such as the nucleosome, chaperonin complex, and the ribosome, suggesting that these core interactions do not change much across the two cell-types (Dataset S11, ESI $\dagger$ ). In contrast, mitochondrial protein complexes were significantly enriched amongst proteins with changing interactions (Table 2 and Dataset S11, ESI $\dagger$ ), suggestive of a substantial rewiring of the mitochondrial interactome between the two cell types. As a result, we hypothesized that mitochondrial activity is likely to be significantly altered between the two cell types. Accordingly, both basal and spare mitochondrial respiratory capacity was significantly reduced in A375M2 cells compared to A375P cells (Fig. 3J). Collectively, these results demonstrate that iCOLA can be used for comparison of functional interactomes between different conditions, and that variations between 
Table 2 Top rewired protein categories between A375P and A375M2 interactomes (from Dataset S11, ESI)

\begin{tabular}{ll}
\hline $\begin{array}{l}\text { Category } \\
\text { database }\end{array}$ & Category name \\
\hline Corum & 55S ribosome, mitochondrial \\
Keywords & Ligase \\
GSEA & RESPIRATORY_ELECTRON_TRANSPORT \\
GOBP & Coenzyme metabolic process \\
GOBP & Cofactor metabolic process \\
GOCC & Mitochondrial matrix \\
Keywords & Mitochondrion \\
Keywords & Transitpeptide \\
GOCC & Mitochondrial part \\
GSEA & TCA_CYCLE_AND_RESPIRATORY_ELECTRON_TRANSPORT \\
GSEA & WONG_MITOCHONDRIA_GENE_MODULE \\
GSEA & MOOTHA_MITOCHONDRIA \\
GOBP & Oxidation-reduction process \\
GSEA & MOOTHA_HUMAN_MITODB_6_2002 \\
GSEA & MITOCHONDRION \\
GOCC & Mitochondrion \\
GSEA & LEE_BMP2_TARGETS_DN \\
GOBP & Small molecule metabolic process
\end{tabular}

interactomes can inform on functional differences between different cellular settings.

\section{COLA is sensitive and specific}

We next assessed the quality of COLA and iCOLA for interactome mapping, by calculating four critical parameters that measure key attributes of a given global protein-protein interaction detection method, as outlined by Vidal and colleagues. ${ }^{27}$ These four parameters are completeness, False Positive Rate (FPR), Sensitivity (Recall), and Precision. Completeness is the measure of the percentage of total possible interactions covered by an assay. FPR is the probability of reporting a false interaction. Sensitivity is the measure of the percentage of all true interactions that are reported by the assay. Finally, Precision measures the percentage of reported interactions which are true. Assay completeness directly corresponds to the number of investigated proteins (i.e. in our case proteins which were identified and quantified in all fractions). Assuming that there are $\sim 22500$ proteins in human genome, the total size of the possible interactome is equal to $22500 \times 22500 / 2=253125000$. In COLA, we identified 1886 proteins, meaning that $1886 \times$ $1886 / 2=1778498$ possible interactions were tested, which is $\sim 1.4 \%$ of the total possible interactome space. This compares similarly with Kristensen et al., SEC-MS method for mining of interactions, ${ }^{12}$ but is less than most $\mathrm{Y} 2 \mathrm{H}$ screens, which cover a larger ORFome space. ${ }^{6,27}$

In a binary interactome analysis, assuming the null-hypothesis (H0) equates to proteins A and B not interacting (false interaction), and alternative hypothesis (H1) to A and B interacting (true interaction), various types of possible interactions can be defined, which are listed in (Table 3).

Assuming that the null hypothesis (H0) in a protein-protein interaction detection assay is no interaction, and the alternative hypothesis (H1) is existence of an interaction, a global binary interactome analysis method can report two types of interaction: true positive (S), and false positive (V). The total number of reported interactions (R) is therefore the sum of $\mathrm{S}$ and $\mathrm{V}$. Conversely, the unreported interactions consist of true (U) and false negatives $(\mathrm{T})$, with the total number of unreported interactions $\left(\mathrm{R}^{\prime}\right)$ consisted of the sum of $\mathrm{U}$ and $\mathrm{T}$. In addition, all true interactions in the interactome space $(\mathrm{M})$, whether reported or not by the method, can be defined as the sum of $\mathrm{S}$ and $\mathrm{T}$. Similarly, all false interactions in the interactome space $\left(\mathbf{M}^{\prime}\right)$, whether reported or not, can be defined as the sum of $V$ and $U$. Finally the total size of the hypothetical interactome space (A) can be defined as the sums of $\mathrm{M}$ and $\mathrm{M}^{\prime}$, or $\mathrm{R}$ and $\mathrm{R}^{\prime}$.

Accordingly, FPR, Sensitivity, and Precision can be defined as a function of these types of interactions:

- Sensitivity $=\mathrm{S} / \mathrm{M}$

- $\mathrm{FPR}=\mathrm{V} / \mathrm{M}^{\prime}$

- Precision $=\mathrm{S} / \mathrm{R}$

CORUM is a highly curated database of well-known protein interactions, which can be regarded as almost 'True'. ${ }^{25}$ To estimate Sensitivity, we can therefore simply calculate the proportion of CORUM interactions that were reported by COLA, for the list of identified input proteins. COLA and iCOLA have a Sensitivity of $\sim 3 \%$ to $6 \%$, depending on the bootstrapping cut-off (Fig. $4 \mathrm{~A}$ ). In comparison, based on CORUM, Kristensen et al.'s SEC-MS method $^{12}$ and Rolland et al.'s Y2H study ${ }^{6}$ had Sensitivities of $\sim 8 \%$ and $\sim 2 \%$, respectively (Fig. $4 \mathrm{~A}$ ). Thus, COLA is roughly similar in terms of its Sensitivity to both of these existing methods.

Estimation of FPR is somewhat tricky as no reference database of false interactions (protein-protein interactions that definitely do not occur) exists. To circumvent this problem, we used two alternative approaches to generate lists of likely to be false interactions. In the first approach, we simply made a list of 200 randomly generated interactions which were not reported to interact with one another in any known protein interaction database (thus at least enriched in false interactions compared to the background), and calculated the percentage of these interactions that were detected by COLA and iCOLA. This method has been utilised by Vidal and colleagues to estimate the FPR of various $\mathrm{Y} 2 \mathrm{H}$ screens, which were reported at 0.5 to $2 \%{ }^{27}$ The downside of such an approach is potential errors that could be introduced due to random sampling. To counteract such bias,

Table 3 Definition of the type of interactions in a given interactome analysis experiment

\begin{tabular}{llll}
\hline & $\mathrm{H}(0)$ is correct & $\mathrm{H}(1)$ is correct \\
\hline Reported interactions & $\mathrm{V}$ (false positives) & $\mathrm{S}$ (true positives) \\
Unreported interactions & $\mathrm{U}$ (true negatives) & $\mathrm{T}$ (false negatives) \\
Sum & $\mathbf{M}^{\prime}$ (total false interactions) & $\mathrm{M}$ (total interactions)
\end{tabular}

Note: $\mathrm{V}+\mathrm{S}=\mathrm{R} ; \mathrm{U}+\mathrm{T}=\mathrm{R}^{\prime} ; \mathrm{V}+\mathrm{U}=\mathrm{M}^{\prime} ; \mathrm{S}+\mathrm{T}=\mathrm{M} ; \mathrm{M}^{\prime}+\mathrm{M}=\mathrm{A}$. 
A

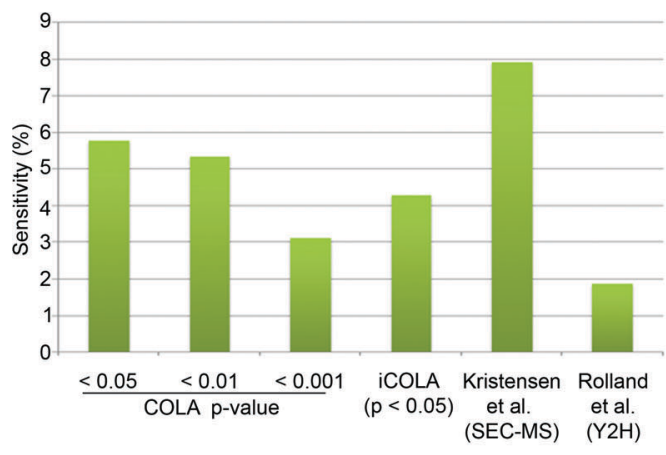

C

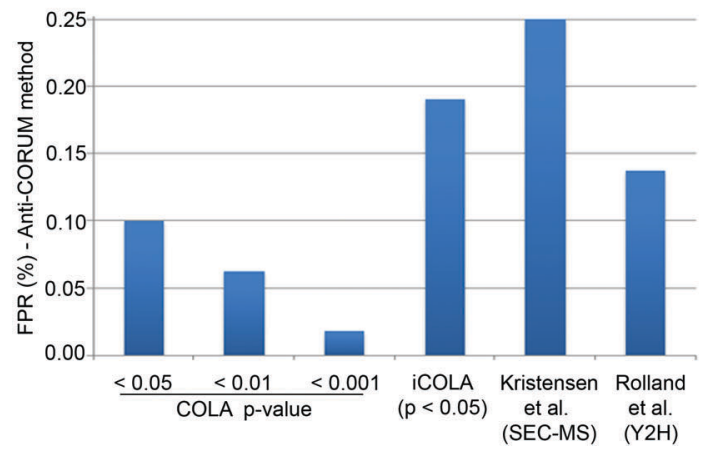

B

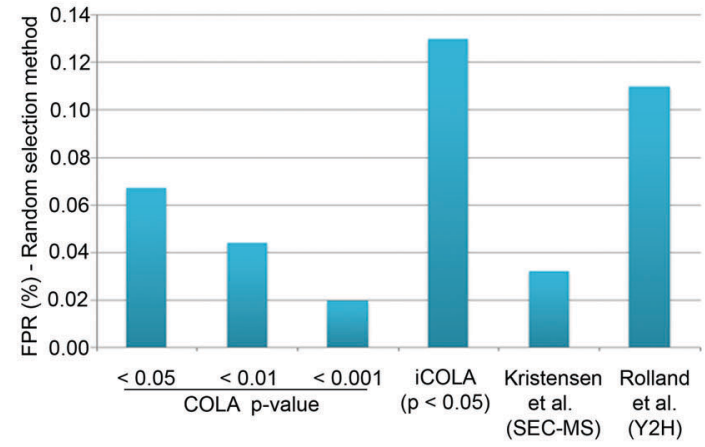

D

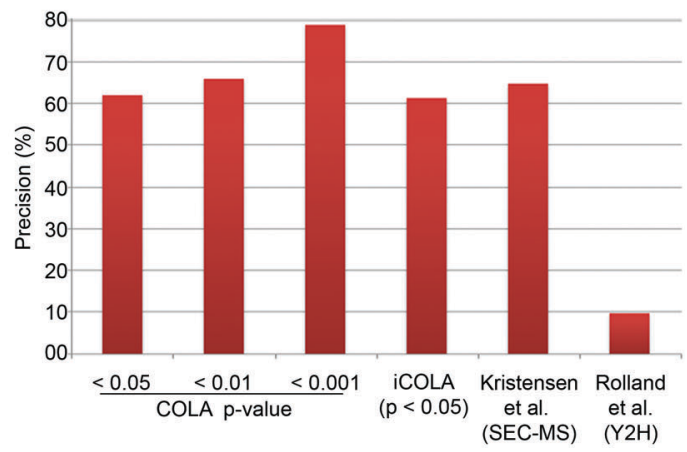

Fig. 4 Analysis of Sensitivity, FPR, and Precision for COLA and iCOLA. (A) Comparison of Sensitivity, also termed Recall, between COLA (at bootstrapping cut off $p$-values of $0.05,0.01$, and 0.001), iCOLA (at cut off $p$-value of 0.05), Kristensen et al.'s SEC-MS method, ${ }^{12}$ and Rolland et al.'s Y2H screen. ${ }^{6}$ Sensitivity was defined as percentage of interactions present in CORUM that were identified by each method. (B) Comparison of FPR between COLA (at bootstrapping cut off $p$-values of 0.05, 0.01, and 0.001), iCOLA (at cut off $p$-value of 0.05), Kristensen et al.'s SEC-MS method, ${ }^{12}$ and Rolland et al.'s $\mathrm{Y} 2 \mathrm{H}$ screen. ${ }^{6} \mathrm{FPR}$ was defined as the percentage of interactions from a list of randomly selected 200 binary interactions not previously reported in any database (see Materials and methods) which were identified by each method. The sampling of 200 unknown interactions was performed 1000 times, and percentage values were averaged and displayed. (C) An alternative comparison of FPR, between COLA (at bootstrapping cut off $p$-values of 0.05 , 0.01 , and 0.001), iCOLA (at cut off $p$-value of 0.05), Kristensen et al.'s SEC-MS method, ${ }^{12}$ and Rolland et al.'s Y2H screen. ${ }^{6}$ FPR was defined as percentage of interactions present in the anti-CORUM dataset (see Materials and methods) that were identified by each method. (D) Comparison of Precision between COLA (at bootstrapping cut off $p$-values of $0.05,0.01$, and 0.001 ), iCOLA (at cut off $p$-value of 0.05 ), Kristensen et al.'s SEC-MS method, ${ }^{12}$ and Rolland et al.'s Y2H screen. ${ }^{6}$ As the total size of the hypothetical interactome space (A), as well as the total number of reported (R) and unreported $\left(R^{\prime}\right)$ interactions are known for each method, the measures of Sensitivity (S/M) and FPR $\left(V / M^{\prime}\right)$ can be used to estimate $S, M, M^{\prime}, T$, and $U$ values. Precision was then calculated as S/R, and displayed as a percentage value. COLA, and iCOLA have comparable or slightly better Precision than Kristensen et al.'s SEC-MS method, but both vastly outperform Rolland et al.'s Y2H.

we repeated our sampling of the 200 unreported interactions a thousand times, calculating the FPR for all of them and averaging the resulting values. Based on this approach, COLA and iCOLA were estimated to have FPR of 0.02 to $\sim 0.1 \%$ depending on the bootstrapping cut-off (Fig. 4B). Using the same estimation strategy, FPR of Kristensen et al.'s SEC-MS was calculated to be $\sim 0.03 \%$ while Rolland $e t$ al.'s Y2H had an FPR of $\sim 0.1 \%$ (Fig. $4 \mathrm{~B}$ ).

A major issue with the aforementioned strategy is that many unreported interactions maybe yet undiscovered true interactions as opposed to false ones, and this is particularly likely if a given protein is not well studied in terms of its interactions, thus not well annotated in interaction databases. So as an alternative approach to generate a library of likely to be false interactions, we used a method based on a strategy recently proposed by Foster and colleagues. ${ }^{31}$ In this method, it is reasoned that as CORUM is a highly curated database of well-known protein interactions, if a given protein is already annotated in CORUM, it is likely that its interactions are better defined, so if two such
CORUM annotated proteins are not reported to interact with each other, they are more likely to be false interactors. Based on this rationale, we created a library of possible false interactions, which we named anti-CORUM (proteins which are listed in CORUM but not known to interact), and used it to estimate the FPR by calculating the percentage of these anti-CORUM interactions that were identified by COLA and iCOLA. Depending on the bootstrapping cut-off stringency, COLA and iCOLA's FPR was estimated at 0.02 to $\sim 0.19 \%$ (Fig. 4C). Using the same strategy, the FPR for Kristensen et al.'s SEC-MS was $\sim 0.25 \%$ while Rolland et al.'s Y2H had an FPR of $\sim 0.14 \%$ (Fig. $4 \mathrm{C}$ ). Thus, the FPR of COLA and iCOLA are better than that of SEC-MS, and better or comparable with $\mathrm{Y} 2 \mathrm{H}$, depending on the bootstrapping cut-off.

Finally, using the anti-CORUM estimated Sensitivity and FPR, we estimated V, S, U, and T values (Table 3), which allowed calculation of Precision for COLA and iCOLA. Depending on the bootstrapping cut off stringency, COLA and iCOLA's Precision were calculated at $\sim 61$ to $79 \%$. In comparison, SEC-MS had a 
Precision of $\sim 65 \%$, while Y2H Precision was $\sim 10 \%$ (Fig. 4D). Thus, while COLA and iCOLA have comparable or slightly better Precision than Kristensen et al.'s SEC-MS, they vastly outperform Rolland et al.'s Y2H screen. Collectively, these results demonstrate that significant similarity in protein localisation signatures can be confidently used to reveal interactions, and that COLA and iCOLA vastly outperform $\mathrm{Y} 2 \mathrm{H}$ in terms of Precision, which is the key measure of assay specificity.

\section{Discussion}

Robust methods that reveal dynamics of functional interactions between proteins on a global scale are crucial for system level understanding of cellular processes. Quantitative proteomics in conjugation with biochemical fractionation by chromatography, ${ }^{11-13}$ is a recent approach that attempts to address the issue of assessing interactome dynamics, but is limited to soluble proteins and can miss on more transient interactions. Here, we present COLA, a global proteomics based strategy that reveals significant co-localisation as a proxy for functional associations. Our approach is not limited to soluble protein complexes and does not solely report biochemical associations. Instead, it robustly matches interacting proteins based on similarity in their multi-dimensional subcellular localisation signatures. In addition, COLA requires significantly less number of proteomics runs per experiment than the previously published chromatography based methods. ${ }^{11,12}$ All subcellular fractionations can be performed in a single day, with proteomics sample preparation and digestion taking two additional days. If the iCOLA approach is used, mass spectrometry runs will only take a few hours, meaning that the whole analysis can be completed in less than a week, including data processing and clustering. This makes iCOLA much faster than any previously published global protein-protein interaction analysis method, and ideal for studying global interactome dynamics. As a proof of principle, we here used iCOLA for such analysis, comparing the interactomes of weakly and highly metastatic isogenic melanoma cells. Our analysis revealed a significant rewiring of the mitochondrial interactome (Table 2), and in line with this observation, the mitochondrial respiratory activity was found to be significantly altered between the two cell-lines (Fig. 3J). The functional significance of this metabolic change with regards to the metastatic potential of the cells remains to be determined.

Although subcellular localisation of proteins has been studied by proteomics before, ${ }^{28,32-37}$ the focus of most of these studies has been on assigning proteins to different organelles rather than revealing protein-protein interactions. Comprehensive subcellular localisation profiling to reveal interactions has been performed by microscopy, using high-throughput fluorescent tagging in combination with high-content imaging. ${ }^{14}$ Also, a related nonmicroscopy based method known as proximity based biotin labelling functions by tagging bait proteins with a promiscuous biotin ligase, which then biotinylates any closely localising proteins in vivo, allowing their subsequent affinity purification and identification of by mass spectrometry. However, similar to
$\mathrm{Y} 2 \mathrm{H}$ or AP-MS, both these approaches suffer from the labour intensive need to tag every target protein in a cell-type of interest, and are prone to potential artefacts caused by the addition of a fluorescent or a biotin ligase tag. In contrast, COLA can be applied to any cell-type, and in a fraction of the time required for other methods. Fractionations, sample preparations, and computational methods used in COLA are all well established, making it a readily available tool to a wide range of biologist across diverse fields. Finally, our benchmarking shows that COLA and ICOLA compare favourably with some of the existing methods of interactome mining in terms of the quality of their interactome data. With regards to their Sensitivity, COLA and iCOLA are comparable with SEC-MS, and perform better than Y2H (Fig. 4A). More importantly, COLA and iCOLA perform comparable or better than SEC-MS in terms of their Precision, while greatly outperforming $\mathrm{Y} 2 \mathrm{H}$ (Fig. 4D). COLA and iCOLA therefore compare favourably with some of the existing global methods for reliable unbiased mining of interactomes.

\section{Conclusions}

Subcellular localisation of a protein is an important determinant of the functional interactions it can form in a given cellular context. COLA uses a quantitative proteomics approach to assess subcellular localisation of proteins on a global scale, and then matches proteins with highly similar subcellular localisation patterns using multi-variate localisation signatures. The rapid nature of COLA, its applicability to almost any cell-type, as well as its accuracy and ease of use for revealing functional interactions on a global scale, renders it highly suitable for assessing functional interactomes across multiple conditions and treatments. We predict that such an approach is likely to have a decisive impact on systems level analysis of functional interactions, as methods to rapidly reveal interactome dynamics on a global scale are desperately needed.

\section{Author contributions}

The study was envisaged by FKM. Experiments were designed by FKM, CB, and CJM. FKM wrote the manuscript. HZS performed all bioinformatics analyses. SK \& RRM assisted with subcellular fractionations. AP assisted with sample preparation of SILAC based COLA samples. CJT and CJ performed sample preparation, TMT labelling, and LC-MS3 analyses of iCOLA samples. SA and GP performed the analysis of mitochondrial flux. All other experiments were performed by FKM.

\section{Conflict of interest}

Authors declare no conflict of interest.

\section{Acknowledgements}

We would like to thank ICR's and Cancer Research UK Manchester Institute's proteomics core facilities for mass spectrometry runs. 
We would also like to thank all Marshall Lab members for their comments and useful discussions. FKM, SK, RRM, AP, and CJM were funded by Cancer Research UK (grant numbers C107/A12057, C107/A10433 \& C107/A16512). CJM was a Gibb Life Fellow of CRUK. HZS and CB were funded by BBSRC project grant BB/J017183/1 and CRUK Programme Foundation Award (C37275/A20146). CJT is funded by a Sir Henry Wellcome Postdoctoral Fellowship (098847/Z/12/Z). CJ is funded by a Cancer Research UK Career Establishment Award (C37293/A12905) and a Cancer Research UK Institute Award (A19258).

\section{References}

1 P. Liberali, B. Snijder and L. Pelkmans, Nat. Rev. Genet., 2015, 16, 18-32.

2 C. Bakal, J. Aach, G. Church and N. Perrimon, Science, 2007, 316, 1753-1756.

3 B. Snijder, P. Liberali, M. Frechin, T. Stoeger and L. Pelkmans, Nat. Methods, 2013, 10, 1089-1092.

4 S. Oliver, Nature, 2000, 403, 601-603.

5 K. Tarassov, V. Messier, C. R. Landry, S. Radinovic, M. M. Serna Molina, I. Shames, Y. Malitskaya, J. Vogel, H. Bussey and S. W. Michnick, Science, 2008, 320, 1465-1470.

6 T. Rolland, M. Tasan, B. Charloteaux, S. J. Pevzner, Q. Zhong, N. Sahni, S. Yi, I. Lemmens, C. Fontanillo, R. Mosca, A. Kamburov, S. D. Ghiassian, X. Yang, L. Ghamsari, D. Balcha, B. E. Begg, P. Braun, M. Brehme, M. P. Broly, A. R. Carvunis, D. Convery-Zupan, R. Corominas, J. Coulombe-Huntington, E. Dann, M. Dreze, A. Dricot, C. Fan, E. Franzosa, F. Gebreab, B. J. Gutierrez, M. F. Hardy, M. Jin, S. Kang, R. Kiros, G. N. Lin, K. Luck, A. MacWilliams, J. Menche, R. R. Murray, A. Palagi, M. M. Poulin, X. Rambout, J. Rasla, P. Reichert, V. Romero, E. Ruyssinck, J. M. Sahalie, A. Scholz, A. A. Shah, A. Sharma, Y. Shen, K. Spirohn, S. Tam, A. O. Tejeda, S. A. Trigg, J. C. Twizere, K. Vega, J. Walsh, M. E. Cusick, Y. Xia, A. L. Barabasi, L. M. Iakoucheva, P. Aloy, J. De Las Rivas, J. Tavernier, M. A. Calderwood, D. E. Hill, T. Hao, F. P. Roth and M. Vidal, Cell, 2014, 159, 1212-1226.

7 E. L. Huttlin, L. Ting, R. J. Bruckner, F. Gebreab, M. P. Gygi, J. Szpyt, S. Tam, G. Zarraga, G. Colby, K. Baltier, R. Dong, V. Guarani, L. P. Vaites, A. Ordureau, R. Rad, B. K. Erickson, M. Wuhr, J. Chick, B. Zhai, D. Kolippakkam, J. Mintseris, R. A. Obar, T. Harris, S. Artavanis-Tsakonas, M. E. Sowa, P. De Camilli, J. A. Paulo, J. W. Harper and S. P. Gygi, Cell, 2015, 162, 425-440.

8 M. Y. Hein, N. C. Hubner, I. Poser, J. Cox, N. Nagaraj, Y. Toyoda, I. A. Gak, I. Weisswange, J. Mansfeld, F. Buchholz, A. A. Hyman and M. Mann, Cell, 2015, 163, 712-723.

9 K. J. Roux, D. I. Kim, M. Raida and B. Burke, J. Cell Biol., 2012, 196, 801-810.

10 A. H. Smits and M. Vermeulen, Trends Biotechnol., 2016, 34, 825-834.

11 P. C. Havugimana, G. T. Hart, T. Nepusz, H. Yang, A. L. Turinsky, Z. Li, P. I. Wang, D. R. Boutz, V. Fong,
S. Phanse, M. Babu, S. A. Craig, P. Hu, C. Wan, J. Vlasblom, V. U. Dar, A. Bezginov, G. W. Clark, G. C. Wu, S. J. Wodak, E. R. Tillier, A. Paccanaro, E. M. Marcotte and A. Emili, Cell, 2012, 150, 1068-1081.

12 A. R. Kristensen, J. Gsponer and L. J. Foster, Nat. Methods, 2012, 9, 907-909.

13 C. Wan, B. Borgeson, S. Phanse, F. Tu, K. Drew, G. Clark, X. Xiong, O. Kagan, J. Kwan, A. Bezginov, K. Chessman, S. Pal, G. Cromar, O. Papoulas, Z. Ni, D. R. Boutz, S. Stoilova, P. C. Havugimana, X. Guo, R. H. Malty, M. Sarov, J. Greenblatt, M. Babu, W. B. Derry, E. R. Tillier, J. B. Wallingford, J. Parkinson, E. M. Marcotte and A. Emili, Nature, 2015, 525, 339-344.

14 Y. T. Chong, J. L. Koh, H. Friesen, S. K. Duffy, M. J. Cox, A. Moses, J. Moffat, C. Boone and B. J. Andrews, Cell, 2015, 161, 1413-1424.

15 F. K. Mardakheh, A. Paul, S. Kumper, A. Sadok, H. Paterson, A. McCarthy, Y. Yuan and C. J. Marshall, Dev. Cell, 2015, 35, 344-357.

16 J. R. Wisniewski, D. F. Zielinska and M. Mann, Anal. Biochem., 2011, 410, 307-309.

17 G. S. McDowell, A. Gaun and H. Steen, J. Proteome Res., 2013, 12, 3809-3812.

18 L. Ting, R. Rad, S. P. Gygi and W. Haas, Nat. Methods, 2011, 8, 937-940.

19 S. Tyanova, M. Mann and J. Cox, Methods Mol. Biol., 2014, 1188, 351-364.

20 S. Tyanova, T. Temu, P. Sinitcyn, A. Carlson, M. Y. Hein, T. Geiger, M. Mann and J. Cox, Nat. Methods, 2016, 13, 731-740.

21 J. Cox and M. Mann, BMC Bioinf., 2012, 13(Suppl 16), S12.

22 R. Suzuki and H. Shimodaira, Bioinformatics, 2006, 22, 1540-1542.

23 A. Franceschini, D. Szklarczyk, S. Frankild, M. Kuhn, M. Simonovic, A. Roth, J. Lin, P. Minguez, P. Bork, C. von Mering and L. J. Jensen, Nucleic Acids Res., 2013, 41, D808-D815.

24 E. G. Cerami, B. E. Gross, E. Demir, I. Rodchenkov, O. Babur, N. Anwar, N. Schultz, G. D. Bader and C. Sander, Nucleic Acids Res., 2011, 39, D685-D690.

25 A. Ruepp, B. Waegele, M. Lechner, B. Brauner, I. DungerKaltenbach, G. Fobo, G. Frishman, C. Montrone and H. W. Mewes, Nucleic Acids Res., 2010, 38, D497-D501.

26 C. J. Tape, S. Ling, M. Dimitriadi, K. M. McMahon, J. D. Worboys, H. S. Leong, I. C. Norrie, C. J. Miller, G. Poulogiannis, D. A. Lauffenburger and C. Jorgensen, Cell, 2016, 165, 910-920.

27 K. Venkatesan, J. F. Rual, A. Vazquez, U. Stelzl, I. Lemmens, T. Hirozane-Kishikawa, T. Hao, M. Zenkner, X. Xin, K. I. Goh, M. A. Yildirim, N. Simonis, K. Heinzmann, F. Gebreab, J. M. Sahalie, S. Cevik, C. Simon, A. S. de Smet, E. Dann, A. Smolyar, A. Vinayagam, H. Yu, D. Szeto, H. Borick, A. Dricot, N. Klitgord, R. R. Murray, C. Lin, M. Lalowski, J. Timm, K. Rau, C. Boone, P. Braun, M. E. Cusick, F. P. Roth, D. E. Hill, J. Tavernier, E. E. Wanker, A. L. Barabasi and M. Vidal, Nat. Methods, 2009, 6, 83-90. 
28 F. M. Boisvert, Y. W. Lam, D. Lamont and A. I. Lamond, Mol. Cell. Proteomics, 2010, 9, 457-470.

29 B. K. Erickson, M. P. Jedrychowski, G. C. McAlister, R. A. Everley, R. Kunz and S. P. Gygi, Anal. Chem., 2015, 87, 1241-1249.

30 E. A. Clark, T. R. Golub, E. S. Lander and R. O. Hynes, Nature, 2000, 406, 532-535.

31 N. E. Scott, L. M. Brown, A. R. Kristensen and L. J. Foster, J. Proteomics, 2015, 118, 112-129.

32 V. K. Mootha, J. Bunkenborg, J. V. Olsen, M. Hjerrild, J. R. Wisniewski, E. Stahl, M. S. Bolouri, H. N. Ray, S. Sihag, M. Kamal, N. Patterson, E. S. Lander and M. Mann, Cell, 2003, 115, 629-640.
33 T. P. Dunkley, R. Watson, J. L. Griffin, P. Dupree and K. S. Lilley, Mol. Cell. Proteomics, 2004, 3, 1128-1134.

34 L. J. Foster, C. L. de Hoog, Y. Zhang, Y. Zhang, X. Xie, V. K. Mootha and M. Mann, Cell, 2006, 125, 187-199.

35 M. W. Trotter, P. G. Sadowski, T. P. Dunkley, A. J. Groen and K. S. Lilley, Proteomics, 2010, 10, 4213-4219.

36 A. Christoforou, C. M. Mulvey, L. M. Breckels, A. Geladaki, T. Hurrell, P. C. Hayward, T. Naake, L. Gatto, R. Viner, A. Martinez Arias and K. S. Lilley, Nat. Commun., 2016, 7, 8992.

37 D. N. Itzhak, S. Tyanova, J. Cox and G. H. Borner, eLife, 2016, 5, e16950. 